\title{
Cardiovascular Manifestations of COVID-19: Insights into a Single-Center Experience
}

\author{
Sara Schukraft ${ }^{1}$ Jean-Luc Magnin ${ }^{1}$ Stéphane Cook $^{1}$ (1) \\ ${ }^{1}$ Department of Cardiology, Hospital and University Fribourg, \\ Fribourg, Switzerland \\ TH Open 2021;5:e329-e334. \\ Address for correspondence Sara Schukraft, MD, Department of \\ Cardiology, University and Hospital Fribourg, Chemin des Pensionnats \\ 2-6, 1708 Fribourg, Switzerland \\ (e-mail: sara.schukraft@yahoo.com).
}

\begin{abstract}
Keywords

- COVID-19

- cardiovascular manifestation

- troponin

Background Since December 2019, an emerging outbreak of novel coronavirus disease 2019 (COVID-19) is caused by the severe acute respiratory syndromecoronavirus-2 (SARS-CoV-2). The aim of the present report is to describe a population with elevated levels of high-sensitive cardiac troponin T (hs-cTnT) and report on their management during the pandemic of COVID-19.

Methods In this retrospective cohort, we collected data from all patients with hs-cTnT levels of $>50 \mathrm{ng} / \mathrm{mL}$ admitted to Fribourg Hospital between February 15, 2020, and April 15, 2020. The primary diagnosis for troponin elevation was recorded. Echocardiographic, electrocardiographic, and coronary angiographic data were analyzed for signs of myocardial ischemia, infarction, or other cardiomyopathies. In-hospital followup was performed for deaths from all causes and for cardiac deaths. Propensity score matching was used in a subgroup analysis to match COVID-19 and non-COVID-19 patients ( $n=21$ per group).

Results Overall, 215 patients with high hs-cTnT levels were enrolled. The median age was 75 [65-83] years and $30 \%$ were women. 21 patients (10\%) were diagnosed with COVID-19. Of these, acute myocardial injury related to COVID-19 was the most commonly described cardiovascular manifestation during the pandemic peak. Median troponin values were not different between COVID-19 patients and non-COVID-19 patients ( 94 vs. $137, p=0.14$ ). The number of cardiological examinations was globally low (echocardiography $51 \%$ and coronary angiography $52 \%$ ) in the context of the pandemic. Patients in the COVID-19 group underwent significantly less echocardiographic examinations ( 19 vs. $55 \%, p \leq 0.01$ ) and coronary angiographies ( 5 vs. $58 \%, p \leq$ 0.01 ) than non-COVID-19 patients. Overall mortality in patient with COVID-19 and elevated troponins was very high, as $38 \%$ of patients died during hospitalization including $14 \%$ for cardiac death. This trend was confirmed in the propensity scorematched analysis.

Conclusion Interpretation of troponins during the COVID-19 pandemic was complicated due to the low number of cardiovascular investigations in this context. Follow-up of patients with COVID-19 and cardiovascular events is important to assess their prognosis and to improve their care.
\end{abstract}

received

September 29, 2021

accepted after revision

April 13, 2021
DOI https://doi.org/

10.1055/s-0041-1731775. ISSN 2512-9465. (c) 2021. The Author(s).

This is an open access article published by Thieme under the terms of the Creative Commons Attribution License, permitting unrestricted use, distribution, and reproduction so long as the original work is properly cited. (https://creativecommons.org/licenses/by/4.0/)

Georg Thieme Verlag KG, Rüdigerstraße 14, 70469 Stuttgart, Germany 


\section{Introduction}

Since December 2019, a large global outbreak of the novel coronavirus disease 2019 (COVID-19) is caused by the severe acute respiratory syndrome-coronavirus-2 (SARS-CoV-2). ${ }^{1}$ The pandemic has been spreading in Switzerland since February $2020 .^{2}$ COVID-19 most commonly manifests with respiratory illness ${ }^{3}$ but cardiovascular involvement has been confirmed. This is due to both the high prevalence of cardiovascular disease in COVID-19 patients and the development of de novo cardiac complications. ${ }^{4}$

Several reports have demonstrated the presence of elevated troponin levels in patients with COVID-19, secondary to acute cardiac lesions including acute myocardial infarction (MI), myocardial injury, acute heart failure, and arrhythmias. ${ }^{5}$ According to the recommendations of the American College of Cardiology (ACC), clinicians should measure troponin only in cases of specific clinical suspicion, as the existence of few prospective data in COVID-19 patients with troponins makes their interpretation complicated. ${ }^{6}$ In response to this review, Chapman and colleagues argued in Circulation that high-sensitivity cardiac troponin is associated with more severe disease and could be an indicator for poorer prognosis in COVID-19 patients. ${ }^{7}$

We sought to describe a population with elevated levels of cardiac troponin T (hs-cTnT), report the cardiological investigations performed during patient's hospitalization and analyze their cardiac diagnosis.

\section{Methods}

\section{Study Population and Data Collection}

We retrospectively included in this analysis all patients admitted to Fribourg Hospital with hs-cTnT $>50 \mathrm{ng} / \mathrm{L}$ between February 15, 2020, and May 8, 2020. The inclusion period was chosen based on the peak pandemic according to in-hospital quality monitoring data. The hs-cTnT levels and other specific biomarkers were performed according to clinical practice. Indication of further cardiac investigations was performed according to the clinicians. A clinical followup was completed for all included patients during the hospital stay. The data collection, analysis, and reporting have been approved by the Institutional Board of the Hospital of Fribourg.

\section{Clinical Endpoints}

The primary diagnosis for troponin elevation was recorded. Laboratory, echocardiographic, electrocardiographic (ECG), and coronary angiographic data were analyzed for signs of myocardial ischemia, infarction, or other cardiomyopathies. Death from any cause and cardiac death were defined using the ARC definition. ${ }^{8}$

\section{Statistical Method}

Categorical variables are reported as count and percentages; continuous variables are reported as mean \pm standard deviation (SD) or median [interquartile range (IQR): 25-75\%]. Normality was assessed by visual inspection of histograms and the com- putation of Q-Q plots. Continuous variables are analyzed using the Student $t$-test or the Wilcoxon rank-sum test according to their distribution. Categorical variables were compared using Chi-square or Fisher's exact test as appropriate. We performed a propensity-matched analysis to adjust for baseline imbalances on the outcomes. In the matching procedure, we used the caliper-matching approach that randomly selected a COVID19 patient with a non-COVID-19 patient from the pool of patients within a caliper of \pm 0.015 on the propensity score. Statistical analyses were performed with Stata version 14.0 (StataCorp LP, College Station, Texas, United States).

\section{Results}

\section{Clinical Characteristics}

During the inclusion period, 215 patients with elevated troponin $\mathrm{T}$ (hs-cTnT) level were enrolled. The median age of the patients was 75 [65-83] years and and 30\% were women. The median length of stay was 6 [3-11] days. The rates of prior hypertension, smoking, diabetes and dyslipidemia were $67,20,22$, and $49 \%$ respectively. - Table 1 shows the baseline characteristics of all patients.

\section{Clinical Investigations}

Patients presented a median troponin value of 133 [77$2,487] \mathrm{ng} / \mathrm{L}$. D-dimer level was available in 43 patients with a median value if 1,293 [618-3,255] ng/mL. The biomarker of inflammation as measured by $\mathrm{C}$-reactive protein (CRP) was recorded in 168 patients with a median value of 27 [35-107] mg/L. Probrain natriuretic peptide (NT-pro-BNP) levels were measured in 71 patients with a median of 3,653 [991-110,053] ng/L. Creatinine median levels were 107 [83144] $\mu \mathrm{mol} / \mathrm{L}$. Of all patients included, 110 (51\%) underwent echocardiography with a median ejection fraction (EF) of $55 \%$ [47-60\%] and 112 (52\%) underwent coronary angiography. Only $1 \%$ of patients with troponins underwent other cardiac investigations, such as cardiac magnetic resonance imaging or coronary computed tomography, during hospitalization. The overall in-hospital death rate was $13 \%$ and cardiac death was $9 \%$ (-Table 2 ). This trend was confirmed in a propensity score-matched analysis. (-Tables $\mathbf{1}$ and $\mathbf{2}$ )

\section{Clinical Diagnosis}

\section{COVID-19 Subgroup}

Clinical diagnosis for troponin elevation was variable. COVID-19 was diagnosed in 21 patients (10\%). Cardiovascular manifestations in 21 patients with COVID-19 infection were analyzed: 14 patients presented with myocardial injury related to COVID-19, 1 patient presented with ST-segment elevation myocardial infarction (STEMI), 1 patient with stress cardiomyopathy, 2 patients with pulmonary embolism (PE), 2 patients with type- $2 \mathrm{MI}$, and 1 patient with congestive heart failure (CHF; - Fig. 1).

\section{Non-COVID-19 Subgroup}

The World Health Organization (WHO) type-1 MI was the most common diagnosis present in $40 \%$ of non-COVID-19 
Table 1 Baseline characteristics

\begin{tabular}{|c|c|c|c|c|c|c|c|}
\hline & \multicolumn{4}{|l|}{ All patients } & \multicolumn{3}{|c|}{ Propensity score-matched patients } \\
\hline & $\begin{array}{l}\text { All patients } \\
(n=215)\end{array}$ & $\begin{array}{l}\text { COVID-19 } \\
(n=21)\end{array}$ & $\begin{array}{l}\text { Non-COVID-19 } \\
(n=194)\end{array}$ & $p$-Value & $\begin{array}{l}\text { COVID-19 } \\
(n=21)\end{array}$ & $\begin{array}{l}\text { Non-COVID-19 } \\
(n=21)\end{array}$ & $p$-Value \\
\hline \multicolumn{8}{|l|}{ Characteristics } \\
\hline Male (\%) & $150(70)$ & $16(76)$ & $134(69)$ & 0.62 & $16(76)$ & $12(57)$ & 0.33 \\
\hline Median age $(\mathrm{y})$ & 75 [65-83] & 77 [73-86] & $75[65-83]$ & 0.04 & $77[73-86]$ & $81[73-86]$ & 0.95 \\
\hline Length of stay (d) & $6[3-11]$ & $8[3-21]$ & $6[2-11]$ & 0.20 & $8[3-21]$ & $7[3-10]$ & 0.55 \\
\hline Hypertension & $143(67)$ & $12(57)$ & $131(68)$ & 0.34 & $12(57)$ & $14(66)$ & 0.75 \\
\hline Smoking & $42(20)$ & $2(10)$ & $40(21)$ & 0.38 & $2(10)$ & $3(14)$ & 1.00 \\
\hline Diabetes & $48(22)$ & $7(33)$ & $41(21)$ & 0.27 & $7(33)$ & $3(14)$ & 0.28 \\
\hline Dyslipidemia & $105(49)$ & $6(29)$ & $99(51)$ & 0.07 & $6(29)$ & $4(19)$ & 0.72 \\
\hline Family history & $21(10)$ & $0(0)$ & $21(11)$ & 0.24 & $0(0)$ & $2(10)$ & 0.49 \\
\hline Previous MI & $35(16)$ & $2(10)$ & $33(17)$ & 0.54 & $2(10)$ & $5(24)$ & 0.41 \\
\hline \multicolumn{8}{|l|}{ Laboratory values } \\
\hline Troponins & 133 [77-320] & 94 [69-194] & 137 [77-369] & 0.14 & 94 [69-194] & 133 [69-252] & 0.36 \\
\hline CRP & 27 [35-107] & 145 [69-238] & $20[5-70]$ & $<0.01$ & 145 [69-238] & $20[6-148]$ & 0.04 \\
\hline Creatinine & $107[83-144]$ & 120 [87-209] & 105 [82-142] & 0.15 & 120 [87-209] & 108 [85-146] & 0.37 \\
\hline D-Dimer & $\begin{array}{l}1,293 \\
{[618-3,255]}\end{array}$ & $4,581[1,355-21,637]$ & $752[505-1,830]$ & $<0.01$ & $\begin{array}{l}4,581 \\
{[1,355-21,637]}\end{array}$ & 711 [421-976] & $<0.01$ \\
\hline NT-pro-BNP & $\begin{array}{l}3,653 \\
{[991-110,053]}\end{array}$ & $3,752[1,726-5,396]$ & $3,650[991-10,053]$ & 0.98 & $\begin{array}{l}3,752 \\
{[1,726-5,396]}\end{array}$ & $7,196[732-11,983]$ & 0.84 \\
\hline
\end{tabular}

Abbreviations: COVID-19, novel coronavirus disease 2019; CRP, C-reactive protein; MI, myocardial infarction; NT-pro-BNP, probrain natriuretic peptide.

Note: Continuous variables are expressed as mean \pm standard deviation, median [interquartile range] or $n$ (\%).

Table 2 In-hospital outcomes

\begin{tabular}{|c|c|c|c|c|c|c|c|}
\hline \multirow[b]{2}{*}{ Investigations } & \multicolumn{4}{|l|}{ All patients } & \multicolumn{3}{|c|}{ Propensity score-matched patients } \\
\hline & $\begin{array}{l}\text { All patients } \\
(n=215)\end{array}$ & $\begin{array}{l}\text { COVID-19 } \\
(n=21)\end{array}$ & $\begin{array}{l}\text { Non-COVID-19 } \\
(n=194)\end{array}$ & $p$-Value & $\begin{array}{l}\text { COVID-19 } \\
(n=21)\end{array}$ & $\begin{array}{l}\text { Non COVID-19 } \\
(n=21)\end{array}$ & $p$-Value \\
\hline Echocardiography & $110(51)$ & 4 (19) & $106(55)$ & $<0.01$ & $4(19)$ & $11(53)$ & 0.05 \\
\hline LVEF & $55[47-60]$ & $50[45-55]$ & $55[47-60]$ & 0.54 & $50[45-55]$ & $60[55-65]$ & 0.13 \\
\hline Coronary angiography & $112(52)$ & $1(5)$ & $111(58)$ & $<0.01$ & $1(5)$ & $12(57)$ & $<0.01$ \\
\hline $\begin{array}{l}\text { Other investigations } \\
\text { (cardiac MRI, coronary CT) }\end{array}$ & $3(1)$ & $0(0)$ & $3(2)$ & 1.00 & $0(0)$ & $0(0)$ & - \\
\hline \multicolumn{8}{|l|}{ In-hospital outcome } \\
\hline In-hospital death & $28(13)$ & $8(38)$ & $20(10)$ & 0.01 & $8(38)$ & $0(0)$ & $<0.01$ \\
\hline In-hospital cardiac death & $19(9)$ & $3(14)$ & $16(8)$ & 0.41 & $3(14)$ & $0(0)$ & 0.23 \\
\hline
\end{tabular}

Abbreviations: COVID-19, novel coronavirus disease 2019; CT, computed tomography; LVEF, left ventricular ejection fraction; MRI, magnetic resonance imaging.

patients including 15\% with STEMI and 23\% with NSTEMI. The incidence of WHO type-2 MI was $21 \%$. We assessed all clinical diagnoses in -Fig. 2 and -Table 3.

\section{COVID-19 versus Non-COVID-19 Patients before Propensity Score Matching}

In the COVID-19 subgroup, patients were older than nonCOVID-19 patients ( 77 vs. 75 years, $p=0.04$; - Table 1). The median CRP amounts ( 145 vs. $20 \mathrm{mg} / \mathrm{L}, p<0.001$ ), as well as the D-dimers ( $4,581 \mathrm{vs.} 752 \mathrm{ng} / \mathrm{mL}, p<0.01$ ), were higher in the COVID-19 group. Troponins were not significantly different in both groups (94 vs. $137 \mathrm{ng} / \mathrm{L}, p=0.14$ ). Only four patients underwent echocardiography and one patient underwent coronary angiography in the COVID-19 group, equivalent to a significantly lower median number of examinations than in the non-COVID-19 group (19 vs. $55 \%, p \leq 0.01$ and 5 vs. $58 \%, p \leq 0.01$, respectively). Overall, mortality in the COVID-19 group was very high, as 38\% of patients died during hospitalization including $14 \%$ for cardiac death. 


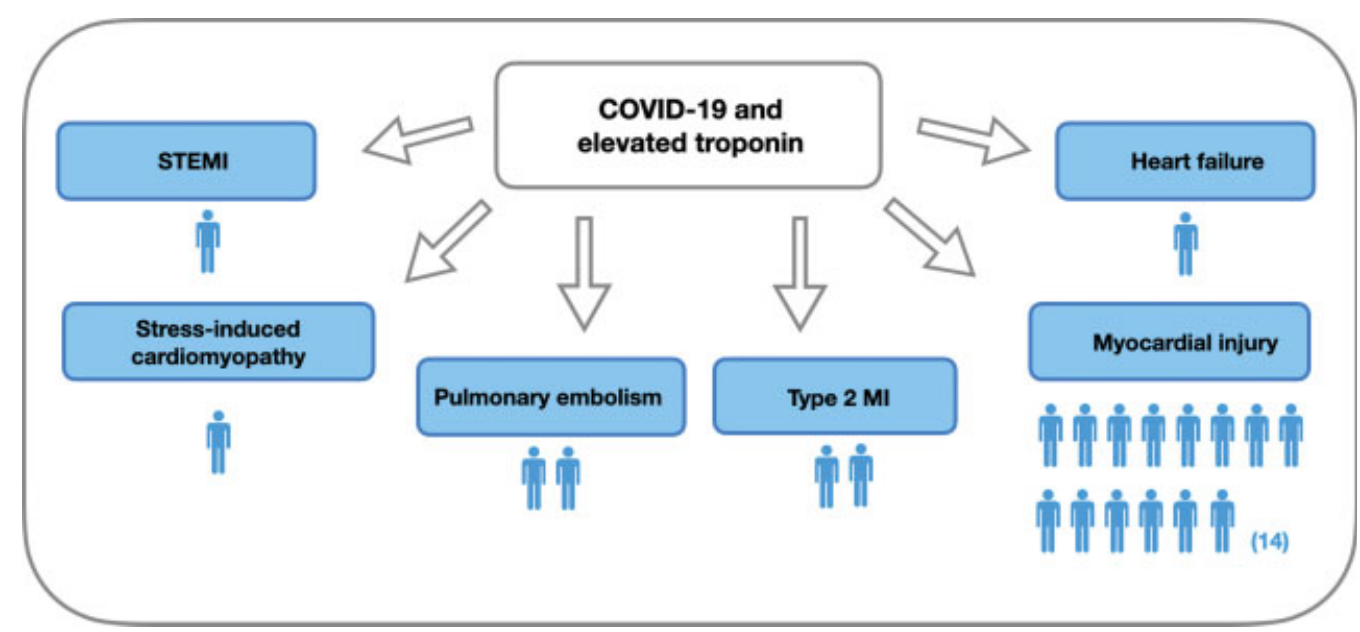

Fig. 1 Cardiovascular manifestations of COVID-19 infection during the pandemic peak. CHF, cardiac heart failure; COVID-19, novel coronavirus disease 2019; MI, myocardial infarction; NSTEMI, non-ST-elevation MI; STEMI, ST-elevation MI.

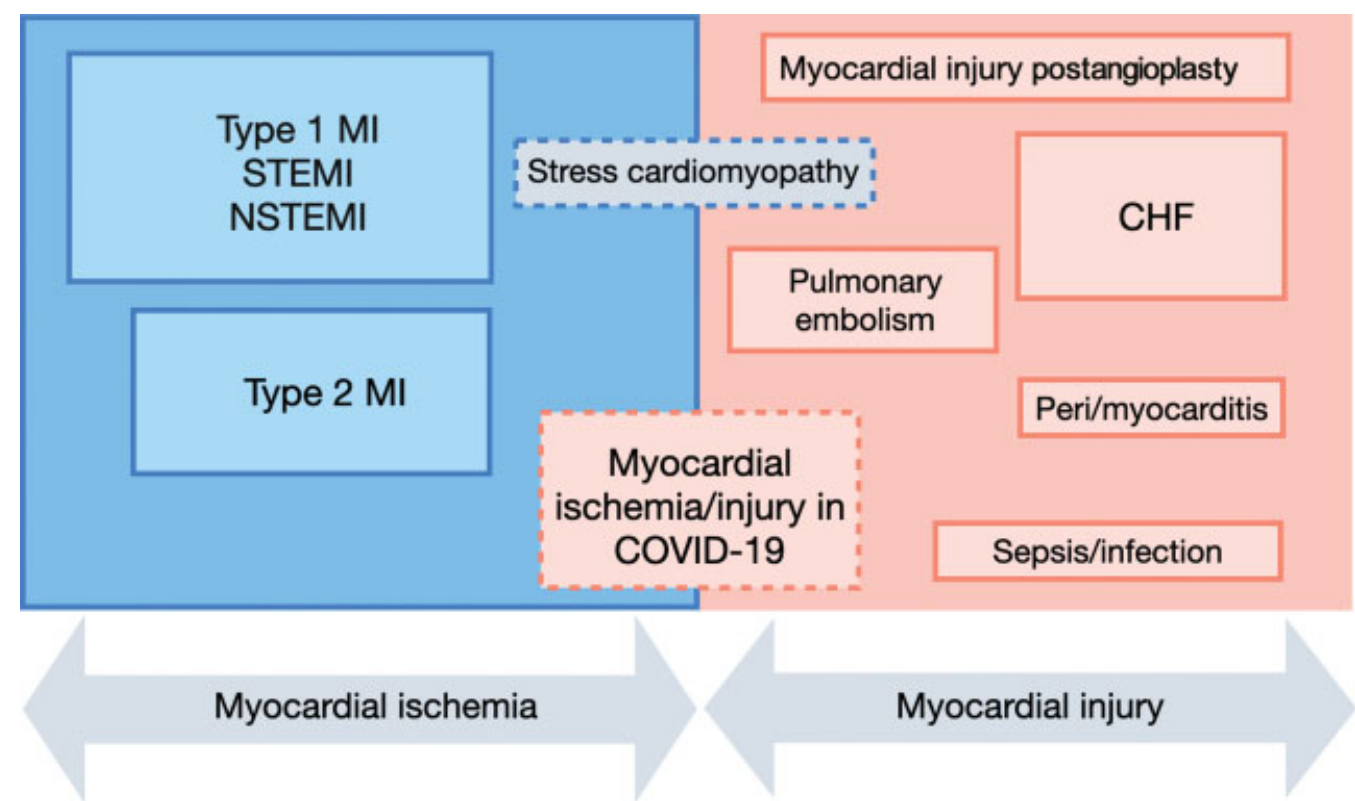

Fig. 2 Clinical evidence of acute myocardial ischemia and myocardial injury during the COVID-19 pandemic. COVID, coronavirus disease; MI, myocardial infarction; STEMI, ST-elevation MI.

\section{COVID-19 versus Non-COVID-19 Patients after Propensity Score Matching}

After propensity score-matching analysis, 42 patients were identified, and baseline characteristics of the patients were well balanced between groups (-Table 1). Troponin levels remained equivalent between the two groups (94 vs. 133, $p=0.36)$. The results confirm a lower number of echocardiographic examinations (19 vs. 53\%, $p=0.05$ ) and coronary angiographies ( 5 vs. $57 \%, p \leq 0.01$ ) between the matched patients (-Table 2 ).

\section{Discussion}

The main findings of this report are as follows: (1) among patients with elevated troponins during the pandemic peak, $10 \%$ were diagnosed with COVID-19; (2) cardiovascular manifestations in patients with COVID-19 infection was varied, ranging from myocardial injury, STEMI, type-2 MI, and stress-induced cardiomyopathy; and (3) in-hospital mortality and cardiac mortality of COVID-19 patients with troponins were high.

\section{Cardiovascular Manifestations of COVID-19 Infection}

This analysis reports a wide and varied panel of cardiac manifestations of COVID-19. Diagnosis of cardiovascular COVID-19 manifestations was performed on a combination of clinical symptoms, specific biomarkers, evidence of new electrocardiographic or echocardiographic abnormalities, or other imaging. Recognition of possible mechanism of cardiovascular manifestations in COVID-19 patients including myocardial injury, plaque rupture or thrombosis (WHO type-1 MI), supply-demand mismatch (WHO type-2 MI), 
Table 3 Clinical diagnosis in non-COVID-19 patients with elevated troponin levels during the pandemic peak

\begin{tabular}{|l|l|}
\hline Clinical diagnose & $\boldsymbol{n}=194$ \\
\hline Type-1 MI & $77(40)$ \\
\hline NSTEMI & $48(23)$ \\
\hline STEMI & $29(15)$ \\
\hline Type-2 MI & $41(21)$ \\
\hline Myocardial injury in renal failure & $27(14)$ \\
\hline Congestive heart failure & $17(9)$ \\
\hline Myocardial injury postangioplasty & $10(5)$ \\
\hline $\begin{array}{l}\text { Myocardial injury in sepsis/infection } \\
\text { (non-COVID-19) }\end{array}$ & $7(4)$ \\
\hline Myocardial injury in PE & $5(3)$ \\
\hline Takotsubo & $3(2)$ \\
\hline Peri-/myocarditis & $3(2)$ \\
\hline Other & $4(2)$ \\
\hline
\end{tabular}

Abbreviations: COVID-19, novel coronavirus disease 2019; MI, myocardial infarction; NSTEMI, non-ST elevation MI; PE, pulmonary embolism; STEMI, ST elevation MI.

Note: Continuous variables are expressed as mean \pm standard deviation, median [interquartile range] or $n(\%)$.

myocarditis, and stress-induced cardiomyopathy is essential for the management and follow-up of these patients.

Acute myocardial injury, defined by an increase in troponins associated or not with electrocardiographic modifications and/or cardiovascular imaging, ${ }^{9-11}$ was the most commonly described cardiovascular complication in COVID-19 in our center during the pandemic peak which is consistent with recent literature. Potential mechanisms of SARS-CoV-2-mediated myocardial injury are direct myocardial injury of the myocardium or secondary to mechanisms related to an acute systemic inflammatory response (cytokine storm). The overall incidence of acute cardiac injury in COVID-19 patients is variable but estimated between 8 and $12 \%$ and is considered an important prognostic marker. ${ }^{3,12}$

\section{Biomarkers}

Troponin values were not different between groups. Although troponins are associated with poorer outcomes in patients with COVID-19 according to recent literature, ${ }^{11}$ it is interesting to note that the degree of elevation did not differ from a group of patients with non-COVID-related cardiac involvement. Since the pattern of troponin elevation is an essential diagnostic factor, this similarity between troponin values is an additional clinical challenge. Shi et al described a case series of 82 patients with cardiac injury and a median troponin troponin value of $190 \mathrm{ng} / \mathrm{L}^{10}$ This higher value of troponin is likely explained by more sever COVID-19 disease in the epicenter of the pandemic with less clinical management experience in the early stages of COVID-19. Finally, recent studies used the 99th percentile as a cut-off point, while our report uses a much higher rule-out threshold (hs-cTnT $>50 \mathrm{ng} / \mathrm{L}$ ) to avoid uncertain diagnosis for patients in the gray zone.

\section{Management Implications}

Of the 21 COVID-19 patients with troponins, 4 patients (19\%) had echocardiography, and 1 patient had a coronary angiography (5\%). The reason for the low number of cardiac examinations in COVID-19 patients with troponins is the initially uncertain interpretation of these biomarkers in the COVID-19 context.

Besides, the presence of strict measures to isolate patients and protect health care workers ${ }^{13}$ during the peak of the pandemic also contributed to the decrease in the number of tests. Indeed, prolonged and close contact during diagnostic tests (e.g., transthoracic echocardiography, transesophageal echocardiography, and coronary angiography) with these patients was limited. This was necessary to minimize routine diagnostic procedures in a setting where health care resources are already stretched and would also expose health care personnel to an increased risk of exposure to infection.

\section{Targeted Cardiac Evaluation}

To optimize the patient's management, targeted cardiac evaluation was indicated in selected patients with COVID19 where the evaluation guided the treatment and prognosis. This approach to cardiac assessment may differ from the standard approach, as it is based on weighing the likelihood of the evaluation-guiding decision-making with nosocomial infection control considerations, in a setting with limited availability of medical resources. The level of precautions taken against COVID-19 were differentiated according to the level of risk based on patient presentation and the type of procedures.

The imaging technique was reevaluated on a patient-bypatient basis, both in terms of diagnostic yield and environmental infectious risk. Routine cardiac imaging in patients with suspected or confirmed COVID-19 was reduced to a strict minimum. The management of patients in our center was largely guided by the ESC recommendations for the diagnosis and management of cardiovascular disease during the COVID-19 pandemic. $^{14}$ Furthermore, emphasis was placed on a proper triage to favor a correct patient assignment based on the infective status and rapid intervention for patients requiring urgent cardiac intervention with adoption of required protective measures.

\section{Potential Long-Term Consequences}

Considering this, careful follow-up of those recovering from the current COVID-19 would be important to understand the long-term impact of this illness and also to protect these patients from future cardiovascular disease.

\section{Limitations}

There are a number of limitations in the present report. Because of the reduced number of invasive and noninvasive imaging during the COVID-19 pandemic, patients were assessed on clinical evidence, and some mechanisms of cardiac injury may have been misdiagnosed. Moreover, this report is limited to intrahospital outcomes and long-term follow-up has not been performed. The presence of COVID-19 infection and 
cardiac involvement does not necessarily evoke a direct causal relationship. Further, laboratory values (such as troponin levels, CRP, creatinine, D-dimer, and NT-pro-BNP) were performed according to clinical practice and were not available for all patients which limits generalization of the laboratory results. Finally, outcome analyses in our study should be interpreted with caution due to the small number of patients. Data from larger population and multicenter data are needed to further confirm the implications of cardiac manifestations in COVID-19.

\section{Conclusion}

This report displays a large panel of possible cardiovascular manifestations of COVID-19. The presumed pathophysiological processes provide a better understanding of these cases but many may have multifactorial etiologies. However, clinical diagnosis was challenged by a reduction of cardiological investigations during the pandemic.

\section{Funding}

The data collection and analysis were supported by an unrestricted grant from the Fonds Scientifique Cardiovasculaire (Fribourg, Switzerland).

\section{Conflict of Interest}

The authors have no conflicts of interest to disclose.

\section{Acknowledgments}

The authors would like to thank Yannick Faucherre for his contribution to the collection of the database record and manuscript revision.

\section{References}

1 Chen L, Liu W, Zhang Q et al. RNA based mNGS approach identifies a novel human coronavirus from two individual pneumonia cases in 2019 Wuhan outbreak. Emerg Microbes Infect 2020;9(01):313-319
2 Federal Office of Public Health. New Coronavirus 2019-nCoV: first confirmed case in Switzerland. Accessed June 10, 2020 at: https:// www.bag.admin.ch/bag/en/home/das-bag/aktuell/medienmitteilungen.msg-id-78233.html

3 Huang C, Wang Y, Li X, et al. Clinical features of patients infected with 2019 novel coronavirus in Wuhan, China. Lancet 2020;395 (10223):497-506

4 Clerkin KJ, Fried JA, Raikhelkar J, et al. COVID-19 and cardiovascular disease. Circulation 2020;141(20):1648-1655

5 Long B, Brady WJ, Koyfman A, Gottlieb M. Cardiovascular complications in COVID-19. Am J Emerg Med 2020;38(07):1504-1507

6 Januzzi JL Jr.American College of Cardiology. Troponin and BNP use in COVID-19. Accessed June 10, 2020 at: https://www.acc. org/latest-in-cardiology/articles/2020/03/18/15/25/troponinand-bnp-use-in-covid19

7 Chapman AR, Bularga A, Mills NL. High-sensitivity cardiac troponin can be an ally in the fight against COVID-19. Circulation 2020; 141(22):1733-1735

8 Cutlip DE, Windecker S, Mehran R, et al; Academic Research Consortium. Clinical end points in coronary stent trials: a case for standardized definitions. Circulation 2007;115(17): 2344-2351

9 Guo T, Fan Y, Chen M, et al. Cardiovascular implications of fatal outcomes of patients with coronavirus disease 2019 (COVID-19). JAMA Cardiol 2020;5(07):811-818

10 Shi S, Qin M, Shen B, et al. Association of cardiac injury with mortality in hospitalized patients with COVID-19 in Wuhan, China. JAMA Cardiol 2020;5(07):802-810. Doi: 10.1001/jamacardio.2020.0950 10.1001/jamacardio.2020.0950

11 Zhang Y, Chen H, Cao B, et al. Clinical course and risk factors for mortality of adult inpatients with COVID-19 in Wuhan, China: a retrospective cohort study. Lancet 2020;135:2033-2040

12 Wang D, Hu B, Hu C, et al. Clinical characteristics of 138 hospitalized patients with 2019 novel coronavirus-infected pneumonia in Wuhan, China. JAMA 2020;323(11):1061-1069

13 World Health Organization. Rational use of personal protective equipment for coronavirus disease (COVID-19): interim guidance, 27 February 2020. Accessed February 27, 2020 at: https://apps. who.int/iris/handle/10665/331215

14 ESC Guidance for the Diagnosis and Management of CV Disease during the COVID-19 Pandemic. Accessed June 10, 2020 at: https://www.escardio.org/Education/COVID-19-and-Cardiology/ ESC-COVID-19-Guidance 